\title{
Magnetic vascular coupling for distal anastomosis in coronary artery bypass grafting: A multicenter trial
}

\author{
Uwe Klima, MD, PhD \\ Volkmar Falk, MD, PhD \\ Michael Maringka ${ }^{a}$ \\ Steffen Bargenda, $\mathrm{MD}^{\mathrm{c}}$ \\ Steven Badack ${ }^{\mathrm{b}}$ \\ Anton Moritz, MD, $\mathrm{PhD}^{\mathrm{c}}$ \\ Friedrich Mohr, MD, $\mathrm{PhD}^{\mathrm{b}}$ \\ Axel Haverich, MD, $\mathrm{PhD}^{\mathrm{a}}$ \\ Gerhard Wimmer-Greinecker, MD, PhD ${ }^{\mathrm{c}}$
}

From the Division of Thoracic and Cardiovascular Surgery, ${ }^{a}$ Medical School, Hannover, Germany; Department of Cardiac Surgery, ${ }^{\mathrm{b}}$ Heart Center University, Leipzig, Germany; and Department of Thoracic and Cardiovascular Surgery, ${ }^{\mathrm{c}} \mathrm{JW}$-Goethe University, Frankfurt, Germany.

Funded by Ventrica, Inc, Fremont, Calif.

Received for publication May 22, 2002; revisions requested Aug 15, 2002; revisions received March 12, 2003; accepted for publication April 10, 2003.

Address for reprints: Uwe Klima, MD, $\mathrm{PhD}$, Department of Thoracic and Cardiovascular Surgery, Hannover Medical School, 30623 Hannover, Germany (Email: klima@thg-mh.hannover.de).

J Thorac Cardiovasc Surg 2003;126: 1568-74

Copyright $\odot 2003$ by The American Association for Thoracic Surgery

$0022-5223 / 2003 \$ 30.00+0$

doi:10.1016/S0022-5223(03)01314-X
Objective: The hand-sewn anastomosis is the "gold standard" for performing coronary artery bypass grafts. However, performing a hand-sewn anastomosis is more demanding and time-consuming when used in less invasive approaches such as small access, totally endoscopic or beating heart surgery. In conjunction with attempts to reduce the surgical trauma of coronary artery bypass grafts by using these less invasive approaches, alternative methods for constructing distal anastomoses should be explored. These data report on predischarge angiographic findings and 30-day clinical follow up of patients who have received a new distal anastomotic device.

Methods: In a multicenter trial, 32 patients (mean age: $65 \pm 9$ years; $85 \%$ men) requiring multivessel coronary artery bypass surgery had 1 of the anastomoses performed using a novel anastomotic technology. The Magnetic Vascular Positioner System was used in 1 of the bypass grafts and the other bypasses were completed by conventional hand-sewn technique. The Magnetic Vascular Positioner System consists of 4 magnetic, gold-plated implants and 2 delivery devices that facilitate the creation of a functional end-to-side anastomosis. A predischarge angiogram was performed to evaluate graft patency.

Results: There were no device-related major adverse events. The application of the Magnetic Vascular Positioner device was successful in 32 of 41 cases (78\%). Nine patients were intended for treatment but did not receive the Magnetic Vascular Positioner System. In 5 of the cases the coronary artery was too small; 1 case had a posterior wall plaque in the target artery; and 3 patients had a nonhemostatic anastomosis after coupling of the port and were subsequently converted to handsewn anastomoses. The median total Magnetic Vascular Positioner anastomotic time was 137 seconds with a range from 65 to 370 seconds. Overall patency rate of the Magnetic Vascular Positioner anastomosis was $93.5 \%$ versus $91.7 \%(P=$ not significant) in hand-sewn grafts. One patient (3.1\%) died due to low cardiac output but had patent grafts at autopsy. One myocardial infarction (3.1\%) occurred the day after a percutaneous transluminal coronary angioplasty of a hand-sewn graft. One prolonged mechanical ventilation $(3.1 \%)$ was required because of pneumonia and adult respiratory distress syndrome.

Conclusions: Magnetic vascular coupling in coronary surgery is safe and effective and has acceptable early patency rates. This new technique may facilitate beating heart and minimally invasive coronary artery bypass grafts. 


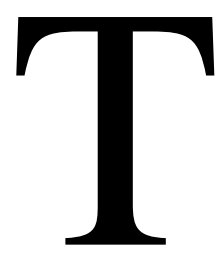

he hand-sewn anastomosis is the "gold standard" in coronary artery bypass grafting (CABG). To reduce the surgical trauma, less invasive approaches (MIDCAB) and beating heart surgery (OPCAB) have been initiated. Because conventional hand-sewn techniques are slow and cumbersome under conditions of limited access, particularly so on the beating heart, alternative methods for constructing the distal anastomosis are being developed. Video-assisted, closed-chest CABG may further complicate the procedure and prolong occlusion time and the ischemic period while tedious anastomoses are being performed. ${ }^{1}$ Numerous attempts have been made to use micromechanical bonding to perform CABG. Scheltes and colleagues $^{2}$ describe 51 devices for the distal anastomosis, categorized into 4 groups: staples, clips, mounting systems, and intraluminal stent structures. ${ }^{3}$ Other sutureless anastomotic concepts such as fibrin and bioglue anastomoses have also been described. ${ }^{4,5}$ Most of the distal anastomotic devices have only been tested in the laboratory and have not found their way into clinical practice. Only a few distal anastomotic devices have been tested clinically utilizing different technical approaches. ${ }^{6-8}$

One goal in coronary surgery is to achieve complete revascularization through a small incision preferably on the beating heart. Currently, few centers are performing these technically demanding and still developmental procedures. ${ }^{9,10}$ Widespread adoption is unlikely without significant advances in technology such as stabilizers and easily deployable and reliable anastomotic devices.

This multicenter clinical trial investigated the safety and efficacy of a novel anastomotic device, the Magnetic Vascular Positioner (MVP) System (Ventrica, Inc, Fremont, Calif) to facilitate the construction of a distal coronary anastomosis.

\section{Methods}

From July 23 to October 16, 2001, thirty-two patients received the MVP device in a multicenter trial conducted at Hannover Medical School, Leipzig Heart Center, and the University Clinic of Frankfurt. The protocol was approved by each center's ethics committee in compliance with the Declaration of Helsinki. ${ }^{11}$ Patient demographics and preimplant characteristics are summarized in Table 1. The MVP study inclusion criteria included subjects with multivessel coronary disease, with the target coronary artery having an upstream stenosis of $\geq 80 \%$, and free of plaque or calcification at the anastomotic site. Subjects excluded from the study had a left ventricular ejection fraction $<30 \%$ or a contraindication to either aspirin or clopidogrel and those patients undergoing emergent $\mathrm{CABG}$, redo $\mathrm{CABG}$, or requiring additional noncoronary procedures. One anastomosis in each patient was performed by means of a magnetic vascular coupling device as described below. All other anastomoses were hand-sewn. Left internal thoracic arteries (LITAs) and saphenous vein grafts (SVGs) were used with the MVP device.
TABLE 1. Patient demographic data and preimplant characteristics

\begin{tabular}{|c|c|c|}
\hline & $n=32$ & MVP Study \\
\hline Age $(y)$, range & & $65 \pm 9(41-80)$ \\
\hline \multicolumn{3}{|l|}{ Gender } \\
\hline Male & 27 & $84.8 \%$ \\
\hline Female & 5 & $15.2 \%$ \\
\hline \multicolumn{3}{|l|}{ CCS class (angina status) } \\
\hline I & 4 & $12.5 \%$ \\
\hline II & 17 & $53.1 \%$ \\
\hline III & 9 & $28.1 \%$ \\
\hline IV & 2 & $6.3 \%$ \\
\hline NYHA class & 1 & $\mathrm{n} / \mathrm{d}$ \\
\hline I & 3 & $9.4 \%$ \\
\hline II & 18 & $56.3 \%$ \\
\hline III & 9 & $28.1 \%$ \\
\hline IV & 1 & $3.1 \%$ \\
\hline \multicolumn{3}{|l|}{ Comorbidities } \\
\hline Hypertension & 23 & $71.9 \%$ \\
\hline Diabetes mellitus & 10 & $31.3 \%$ \\
\hline Hypercholesterolemia & 15 & $46.9 \%$ \\
\hline Smoker & 15 & $46.9 \%$ \\
\hline Family history of coronary disease & 10 & $31.3 \%$ \\
\hline Renal failure & 2 & $6.3 \%$ \\
\hline Chronic obstructive pulmonary disease & 4 & $12.5 \%$ \\
\hline Peripheral vascular disease & 8 & $25 \%$ \\
\hline Cerebrovascular disease & 5 & $15.6 \%$ \\
\hline Other major morbidity & 7 & $21.9 \%$ \\
\hline \multicolumn{3}{|l|}{ Hyperlipoproteinemia $(\mathrm{n}=2)$} \\
\hline \multicolumn{3}{|l|}{ Hyperuricemia } \\
\hline \multicolumn{3}{|l|}{ Obesity (BMI > 30) } \\
\hline \multicolumn{3}{|l|}{ Hyponatremia } \\
\hline \multicolumn{3}{|l|}{ Pacemaker } \\
\hline Pulmonary embolis & & \\
\hline
\end{tabular}

CCS, Canadian Cardiovascular Society angina classification; NYHA, New York Heart Association classification; $n / d=$ no data; $B M I$, body mass index; MVP, Magnetic Vascular Positioner. Results are mean \pm standard deviation.

\section{Preoperative and Postoperative Anticoagulation Regimen}

Twelve to 24 hours preoperatively patients received a single loading dose of $500 \mathrm{mg}$ aspirin and $300 \mathrm{mg}$ clopidogrel (Plavix; Sanofi Synthelabo GmbH, Berlin, Germany). On the day of operation no concomitant anticoagulation therapy was administered except for heparin for cardiopulmonary bypass. On the first postoperative day patients started $75 \mathrm{mg}$ of clopidogrel daily for 12 weeks and a chronic course of $100 \mathrm{mg}$ of aspirin daily.

\section{Operative Procedure}

Standard techniques for induction and maintenance of anesthesia during the procedure were used in all 3 centers. Twenty-six patients were operated on using standard cardiopulmonary bypass techniques and cardiac arrest. Heparin (350 U/kg) was administered initially; activated clotting time levels were thereafter targeted to remain above 400 seconds. Six patients had an off-pump beating heart procedure with mechanical stabilization using the Axius stabilization system (Guidant, Santa Clara, Calif). Aprotinin 


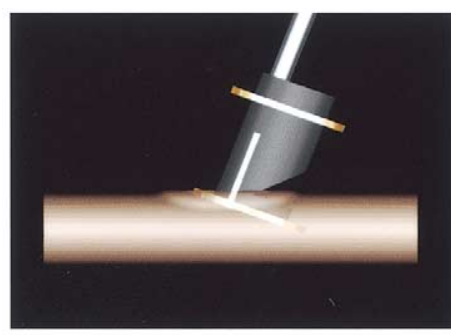

1. Insertion

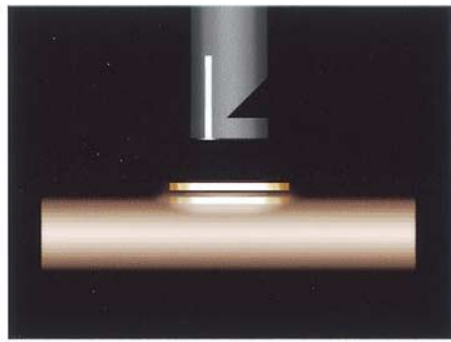

3. Device

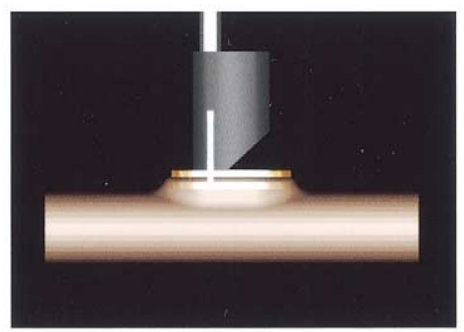

2. Deployment

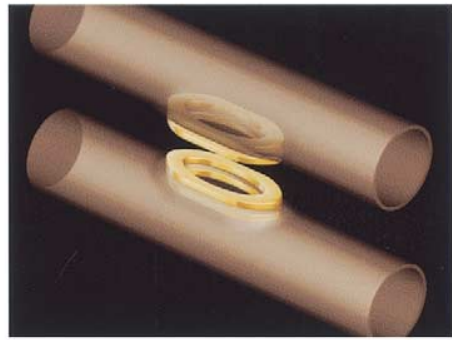

4. Connection

Figure 1. The Magnetic Vascular Positioner System consists of 4 magnetic implants and 2 delivery systems for completion of an anastomosis. Illustrations show the stepwise completion of an MVP anastomosis. See text for detailed information.

(Trasylol; Bayer, Leverkusen, Germany) or epsilon amino capron acid was not routinely used.

\section{Target Vessel and Intention to Treat}

The target vessel and site of anastomosis was chosen intraoperatively based on the surgeons' personal subjective decision. The anastomotic site was required to be free of plaque and to have a minimum intraluminal diameter of $2.0 \mathrm{~mm}$. Intention to treat was defined as intraoperative decision to attempt to perform an MVP anastomosis.

\section{Description of Device}

The MVP System consists of 4 magnetic implants and 2 delivery systems for completion of an anastomosis. A pair of magnetic, elliptically shaped, gold-plated implants with an open area of 8.1 $\mathrm{mm}^{2}$ is placed into a $5.0 \mathrm{~mm}$ longitudinal incision near the end of a ligated graft vessel to create an anastomotic port (Figure 1). One magnetic implant lies just within the graft lumen at the incisional site (intravascular magnetic implant). The other is held in place on the external surface of the vessel by mutual magnetic attraction, precisely above the first implant (extravascular magnetic implant). The implants hold the elliptically shaped anastomotic port open. The second pair of magnetic implants forms an identical port in the coronary artery and is introduced in the same manner as was done at the graft port. The 2 ports formed by the magnetic implant pairs are then brought together, allowing the magnetic attraction of the 2 pairs to maintain apposition and form a reliable side-to-side anastomosis (functional end-to-side). The distal end of the bypass was either clipped or tied off. In addition, 2 to 4 tacking sutures were placed according to the protocol to secure the position of the MVP graft.

\section{Angiographic and 30-Day Postoperative Clinical Evaluation}

Patency was evaluated angiographically prior to hospital discharge. Bypass grafts were graded as either patent or not patent based on evidence of flow going from the graft into the target vessel. The 30-day clinical follow-up included a physical examination, an assessment of angina status, and a determination of the presence or absence of a major adverse cardiac events: death, myocardial infarction, or reintervention.

\section{Statistical Analysis}

Data are expressed as mean \pm standard deviation. In addition, $95 \%$ confidence intervals were calculated to facilitate comparison of patency rates between groups. After testing for normal distribution the paired Student $t$ test was used for intragroup comparison of the Canadian Cardiovascular Society (CCS) angina class; $P<.05$ was considered significant.

\section{Results}

Forty-one patients were enrolled in the study. Of these, 32 patients $(78 \%)$ received the device and were followed. In 


\section{TABLE 2. Patient implant characteristics}

\begin{tabular}{lrc}
\hline & $\mathbf{n}=\mathbf{3 2}$ & MVP study \\
\hline MVP graft vessel & & \\
SVG & 23 & $71.9 \%$ \\
LITA & 9 & $28.1 \%$ \\
MVP target vessel & & \\
Mid RCA & 1 & $3.1 \%$ \\
Distal RCA & 5 & $15.6 \%$ \\
Right PDA & 1 & $3.1 \%$ \\
Right PLA & 2 & $6.3 \%$ \\
Mid LAD & 7 & $21.9 \%$ \\
Distal LAD & 5 & $15.6 \%$ \\
1st diagonal & 4 & $12.5 \%$ \\
1st obtuse Marginal & 4 & $12.5 \%$ \\
2nd obtuse Marginal & 1 & $3.1 \%$ \\
Left PLA & 1 & $3.1 \%$ \\
Ramus & 1 & $3.1 \%$ \\
Procedure time (minutes), & & $221 \pm 118$ (160-840) \\
$\quad$ range & & \\
CPB time (minutes), range & & $92 \pm 16$ (66-124) \\
Cross clamp time (minutes), & & $46 \pm 19(23-116)$ \\
$\quad$ range & & \\
MVP connection time & & \\
$\quad$ (seconds), range & & \\
Total median MVP & & \\
$\quad$ deployment and & & \\
$\quad$ connection time (seconds) & & \\
\hline
\end{tabular}

MVP, Magnetic Vascular Positioner; SVG, saphenous vein graft; LITA, left internal thoracic artery; $R C A$, right coronary artery; $P D A$, posterior descending artery; $P L A$, posterolateral artery; $L A D$, left anterior descending artery.

*Total crossclamp time for on-pump patients only $(n=26)$.

the 32 patients undergoing multivessel coronary revascularization, a total of 32 MVP and 74 hand-sewn anastomoses were performed. Clinical data for the MVP anastomoses are summarized in Table 2. A representative postoperative angiogram of an MVP LITA graft is shown in Figure 2. There were no intra- or postoperative adverse events related to the use of the MVP system.

In 3 patients the MVP anastomosis had to be redone due to excessive tissue within the lumen of the anastomotic area (graft or coronary port). The study design targeted $<10 \%$ tissue in the orifice of the MVP port on either the coronary artery or graft. In all 3 cases the magnet pairs could be easily removed without any apparent trauma to the target vessels. In all of these cases, the MVP was redeployed with a successful anastomosis being created and all were patent as demonstrated in the predischarge angiogram.

\section{Major Adverse Events}

There was 1 death (3.1\%) in this series, unrelated to the device. The patient had a hand-sewn LITA graft to the left anterior descending artery (LAD), a hand-sewn SVG to the second obtuse marginal branch (OM), and an MVP SVG to the intermediate branch. After an initially uneventful oper-

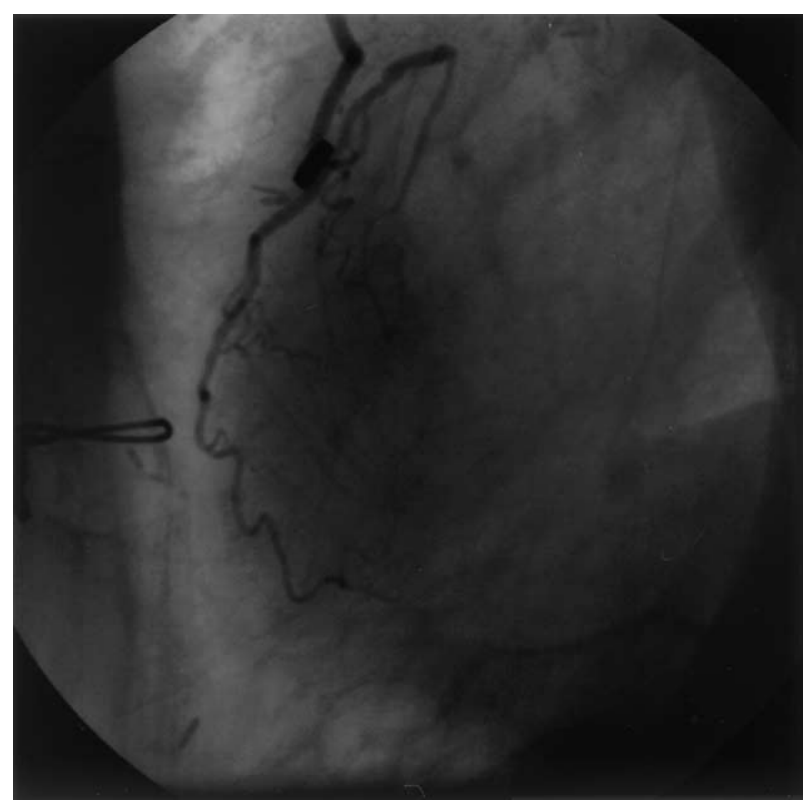

Figure 2. Postoperative angiogram of MVP anastomosis of a LITA to LAD graft.

ation the patient's electrocardiogram (ECG) showed ischemia of the anterior wall and septum. Cardiac enzymes were within normal limits. The patient was reexplored and no graft occlusions were found. The cause of death has been classified as low cardiac output syndrome. At autopsy all grafts were confirmed as patent. No histological studies of the MVP device were performed, as the device was not explanted at the time of autopsy.

One patient $(3.1 \%)$ suffered a myocardial infarction with positive changes in ECG leads II, III, and aVF. This patient had a hand-sewn LITA graft to the LAD, a hand-sewn SVG to the first OM, and an MVP SVG to the posterior descending artery. At the predischarge angiogram a stenosis was detected in the midsection of the SVG to the OM1 handsewn graft. Though the patient was asymptomatic, a percutaneous transluminal coronary angioplasty was performed in the stenosed graft. The following day, the patient sustained a myocardial infarction. The patient was hemodynamically stable and did not undergo any further reintervention. This event was not device related.

One patient $(3.1 \%)$ required prolonged mechanical ventilation due to pneumonia and adult respiratory distress syndrome. The patient was discharged to rehabilitation for ventilator weaning.

\section{Other Adverse Events}

There were 3 (9.3\%) reexplorations for bleeding. One patient sustained a cardiac tamponade 2 hours after operation and was returned to the operating room. No source of bleeding could be identified; there were no anastomotic 
leaks. One patient was reexplored for bleeding on the ninth postoperative day. No source of bleeding could be identified. The third patient experienced bleeding from a side branch of a radial artery graft leading to a hand-sewn anastomosis. These 3 patients reached the 30-day clinical end point and continued to be followed. There were 2 $(6.1 \%)$ transluminal coronary interventions in this series: 1 patient with an occluded MVP anastomosis and 1 patient with a stenosed vein graft. Both patients were asymptomatic and were diagnosed during the routine predischarge angiogram. The interventions were performed at the time of the diagnostic procedure. The patient with the MVP occlusion was treated by percutaneous stenting of the native coronary artery (SVG to OM1). The patient with the stenosed handsewn graft had a percutaneous angioplasty procedure to the graft (SVG to OM1).

There was $1(3.1 \%)$ transient ischemic attack prior to hospital discharge. The patient's cerebral computed tomography showed no morphologic changes. Recovery was complete.

\section{Intention to Treat}

A successful anastomosis was performed in $78 \%$ of cases (32/41). In addition to the 32 patients who received an MVP anastomosis, 9 patients were classified as intention to treat patients. Four of the 9 patients initially received the MVP implant but were converted to a hand-sewn anastomosis for the following reasons: posterior plaque formation in $1 \mathrm{pa}-$ tient and 3 patients had inadequate hemostasis at the anastomotic site after the coupling due to insufficient tissue capture within the magnetic implants. This is an error in deployment technique and was noted upon immediate test flushing of the anastomosis. In the other 5 patients the magnets were not inserted into the coronary artery due to improper judgment of the target vessel diameter. The target vessels appeared to be larger from the outside; however, after incising the vessels, the internal diameter was unacceptable for inserting the magnets due to sclerotic narrowing.

\section{Angiographic Follow-up}

A total of 32 patients (32 MVP and 74 hand-sewn grafts) received the MVP device. Of these, 31 patients (1 death) had a postoperative coronary angiogram prior to hospital discharge (mean of $6.3 \pm 5.4$ days). Thirty-one MVP and 72 hand-sewn anastomoses were evaluated. The overall patency rate was $93.5 \%$ (29 of 31) in the MVP group versus 91.7\% (66 of 72) in the hand-sewn group ( $P=$ not significant). In addition to the occluded grafts, 3 hand-sewn grafts were found to have stenoses $>50 \%$. There was a $100 \%$ ( 9 of 9) patency rate in left internal thoracic to LAD MVP anastomoses.

\section{Thirty-day Clinical Follow-up}

There was significant clinical improvement at the 30-day evaluation with a mean CCS status of $0.37 \pm 0.5$ at 30 days postoperatively versus $2.3 \pm 0.8$ preoperatively $(P<$ .0001). Angina modification, as assessed by CCS classification, measured a secondary efficacy end point. On the preprocedure evaluation, $81.2 \%$ of assessments indicated a class II or class III angina status, with the remainder falling into class I or class IV. At the 30 -day evaluation $63.3 \%$ of patients were evaluated as class 0 and $36.7 \%$ as class I.

No new major adverse events occurred within the 30-day follow-up period. Minor adverse events included new onset of atrial fibrillation in 2 patients $(6.5 \%)$, pneumonia in 1 patient $(3.2 \%)$, and leg wound infection in 1 patient $(3.2 \%)$. The overall minor adverse event rate was $13.1 \%$. Of the 31 surviving patients, ECGs demonstrated no new myocardial infarction or Q-wave changes during a mean follow-up of 32 days.

\section{Discussion}

This study demonstrates the safety and efficacy of a novel, distal anastomotic connector. In addition, this study represents the first worldwide clinical use of this unique technology in humans. Although several proximal anastomotic devices have been tested, clinically only a few clinical studies about devices for the much more technically demanding distal anastomosis have been published. ${ }^{7,8}$ The authors describe several techniques for performing the anastomoses between SVGs or internal thoracic arteries and coronary arteries including the use of clips, glue, or stentlike applicators. The concept of magnetic coupling is vastly different from other contemplated micromechanical methods and has several advantages as compared with handsewn anastomosis. It is simple, easy to use, and offers the benefit of a quick and repeatable anastomosis, which may facilitate less invasive procedures. The median time for MVP deployment and connection was 137 seconds. This included the time to deploy the graft port, the time to deploy the coronary port, and the time to couple the 2 together. As each vessel is prepared prior to completion of the anastomosis, the surgeon is able to inspect and confirm a consistent and uniform port opening prior to completing the anastomosis. Although the anastomotic times were very short with the MVP, it is anticipated that these will be reduced even further with more frequent use of the device. In 26 of the 32 patients, an MVP anastomosis was performed on the arrested heart. This approach was chosen to evaluate the device while not introducing new variables. However, after successful application in the stopped heart environment we also successfully applied the magnetic vascular positioner in 6 beating heart cases. Going forward, we expect that the MVP will be more widely used in beating heart and less 
invasive approaches as it may limit ischemic times associated with conventional hand-sewn anastomoses.

There was not an appreciable learning curve associated with handling of the device. Only a few deployments in the animal model were needed to become familiar with the different delivery aspects of the MVP system. An important part of the MVP procedure is the arteriotomy. A targeted arteriotomy length of 4.5 to $5.0 \mathrm{~mm}$ was desired. There is approximately $1.0 \mathrm{~mm}$ of variability that can be accommodated by the device. No cases had to be aborted due to improper incision length.

As described in Table 2 the MVP device could be applied to all 3 coronary arteries and their side branches. Both LITAs and SVGs were used as bypass conduits for the MVP anastomosis. The radial artery was not used with the MVP as the preclinical testing had been done on internal thoracic arteries and SVGs only. However, the device appears to be compatible with radial arteries based on our evaluation of remnant pieces of radial artery during the clinical investigation. Consequently, the MVP system seems to be applicable to all target vessels and graft conduits.

\section{Limitations of Study and Device}

This iteration of the device was targeted for coronary vessels with an intraluminal diameter $\geq 2.0 \mathrm{~mm}$. Of the vessels treated with the MVP, 30 were within this range and 2 were below. At the moment only 1 size magnet is available, limiting the applicability to target vessels with smaller diameters. A second generation of downsized magnets is currently being evaluated with early results in animals demonstrating similar patency rates in target vessels as small as $1.5 \mathrm{~mm}$. A device for vessels smaller than $1.0 \mathrm{~mm}$ will not likely be needed due to questionable perfusion benefit as well as poor patency rates in these coronary targets. ${ }^{12}$ The current version of the device with a straight delivery system limits its flexibility on the posterolateral wall. Even though an anastomosis could be performed in the circumflex artery area, an angled delivery system will certainly facilitate MVP placement in posterolateral vessels. For purposes of this study, anastomotic target sites with diffuse disease, calcification, and visible plaques were avoided. However, we were routinely able to find an acceptable target site. In cases where an ideal deployment was not achieved (eg, too much tissue in the lumen), the device could be easily removed with no apparent trauma to the tissue. Following removal of the MVP, the device could be redeployed or a hand-sewn anastomosis could be performed if desired. This bailout feature helped to provide an extra level of comfort when performing an anastomosis with a new, mechanical device.

\section{Angiographic Results}

The predischarge angiographic patency of $93.5 \%$ for the MVP system was very encouraging and compares favorably with other CABG trials with angiographic end points at this time point. ${ }^{13,14}$ Of particular note was the $100 \%$ patency rate of the MVP in the LITA-LAD position. In the group of hand-sewn anastomosis 3 grafts had a stenosis $>50 \%$. These anastomoses were classified as patent in our analysis. The reason for doing so was that no quantitative coronary flow analysis was performed and the results could not be objectively assessed in either group. Due to the radiopacity of the MVP system, a stenosis in the MVP anastomosis could not be assessed angiographically, and hence an anastomosis was classified as patent when good runoff could be demonstrated.

\section{Anticoagulation Regimen}

Preoperative antiplatelet therapy was instituted because the MVP system has exposed foreign material in the blood flow path. Furthermore, it is yet unknown in humans if the application of a magnet into the coronary arteries may cause similar problems as seen after stent application with intimal hyperplasia formation and restenosis. The combination of aspirin and clopidogrel was chosen for several reasons. Clopidogrel, a platelet adenosine diphosphate receptor antagonist, has been shown to inhibit platelet aggregation and to prevent the recurrence of ischemic events more effectively than aspirin. This is due to a short-lasting metabolite produced by activation of hepatic cytochrome P450-1A. ${ }^{15}$ Recent experimental data demonstrated that the antiaggregating, antithrombotic, and antiproliferative activity of clopidogrel was potentiated by aspirin. ${ }^{16}$ Patients received preoperative loading dose of clopidogrel and aspirin to obtain the protective antithrombotic effect immediately at the end of the operation. Prior to commencing clinical trials with the MVP system, extensive preclinical testing was performed. Acute and chronic animal studies (swine and ovine) were performed to demonstrate acceptable performance of the device. In yet unpublished experimental data a patency rate of $97 \%$ after a 1-month follow-up period was demonstrated using this antiplatelet regimen. Additionally, animals killed at 3 and 6 months were found to have similar patency rates and demonstrated a fully healed and stable tissue layer that lined the lumen and covered the magnets. Histological analysis showed a smooth, uniform endothelial covering, lining the entire lumen. ${ }^{17}$ Hence, we decided to administer a combination of clopidogrel and aspirin for 12 weeks, and thereafter a single antiplatelet therapy with aspirin only.

Prior to surgery, many patients are currently taking antiplatelet drugs for various reasons. These include routine antiplatelet administration following prophylactic loading in the catheterization laboratory, stent implantation, and clopidogrel therapy for multivessel disease. To minimize potential thrombotic problems we thought it prudent and reasonable to use full antiplatelet therapy. Since commence- 
ment of this trial, recent studies have been published regarding reexploration rates in $\mathrm{CABG}$ patients on antiplatelet therapy. Hongo and colleagues ${ }^{18}$ reported a $6.8 \%$ reoperation rate and Yende and colleagues ${ }^{19}$ reported a rate of $9.8 \%$. We now have additional animal data to support using reduced antiplatelet dosage in subsequent trials.

\section{Conclusion}

This study demonstrates the efficacy and safety of a novel, distal anastomotic connector by means of magnetic vascular coupling. The study also represents the first worldwide clinical application in humans of this new technique. Early safety and patency of the MVP system appears encouraging. The device is easy to use and should be adaptable to OPCAB, MIDCAB, and TECAB procedures. Additional studies and long-term follow-up will be required to evaluate the long-term patency of the device.

\section{References}

1. Mack MJ, Acuff TE, Casimir-Ahn H, Lönnn UJ, Jansen EW. Video assisted coronary bypass grafting on the beating heart. Ann Thorac Surg. 1997;63:S100-3.

2. Scheltes JS, Heikens M, Pistecky PV, van Andel CJ, Borst C. Assessment of patented coronary end-to-side anastomotic devices using micromechanical bonding. Ann Thorac Surg. 2000;70:218-21.

3. Calafiore AM, Bar-El Y, Vitolla G, et al. Early clinical experience with a new sutureless anastomotic device for proximal anastomosis of the saphenous vein to the aorta. J Thorac Cardiovasc Surg. 2001;121: 854-8.

4. Detweiler MB, Detweiler JG, Fenton J. Sutureless and reduced structure anastomosis of hollow vessels with fibrin glue: a review. J Invest Surg. 1999;12:245-62.

5. Gundry SR, Black K, Izutani H. Sutureless coronary artery bypass with biologic glued anastomoses: preliminary in vivo results. $J$ Thorac Cardiovasc Surg. 2000;120:473-7.

6. Niinami H, Takeuchi Y, Ichiawa S, et al. Coronary artery bypass- grafting using interrupted anastomosis with the U-clip. Kyobu Geka. 2001;54:1003-6.

7. Tozzi P, Stumpe F, Ruchat O, Marty B, Corno AF, von Segesser LK. Preliminary clinical experience with the heartflo anastomosis device. Thorac Cardiovasc Surg. 2001;49:279-82.

8. Eckstein FS, Bonilla LF, Meyer B, Berg TA, Neidhart PP, Schmidli J, Carrel TP. Sutureless mechanical anastomosis of a saphenous vein graft to a coronary artery with a new connector. Lancet. 2001;357: 931-2.

9. Falk V, Diegeler A, Walther T, et al. Total endoscopic coronary artery bypass grafting. Eur J Cardiothorac Surg. 2000;17:38-45.

10. Dogan S, Aybek T, Westphal K, Mierdl S, Moritz A, WimmerGreinecker G. Computer-enhanced totally endoscopic sequential arterial coronary artery bypass. Ann Thorac Surg. 2001;72:610-1.

11. World Medical Association, Inc. The Helsinki Declaration: Ethical Principles for Medical Research Involving Human Subjects. October $2000<$ http://www.wma.net/e/policy/17-c_e.html $>$.

12. Paz MA, Lupon J, Bosch X, Pomar JL, Sanz G. Predictors of early saphenous vein aortocoronary bypass graft occlusion. The GESIC Study Group. Ann Thorac Surg. 1993;56:1101-6.

13. Okawa Y, Baba H, Hashimoto M, et al. Comparison of standard coronary artery bypass grafting and minimally invasive direct coronary artery bypass grafting. Early and mid-term result. Jpn J Thorac Cardiovasc Surg. 2000;48:725-9.

14. Wiklund L, Johansson M, Bugge M, Radberg LO, Brandup-Wognsen G, Berglin E. Early outcome and graft patency in mammary artery grafting of left anterior descending artery with sternotomy or anterior minithoracotomy. Ann Thorac Surg. 2000;70:79-83.

15. David JL, Limet R. Antiplatelet activity of clopidogrel in coronary artery bypass graft surgery patients. Thromb Haemost. 1999;82:141721.

16. Herbert JM, Dol F, Bernat A, Falotico R, Lale A, Savi P. The antiaggregating and antithrombotic activity of clopidogrel is potentiated by aspirin in several experimental models in the rabbit. Thromb Haemost. 1998;80:512-8.

17. Adams DH, Filsoufi F, Aklog L, et al. Automated distal coronary bypass using a novel magnetic coupler. American Association for Thoracic Surgery 82nd Annual Meeting, Washington, DC, May 5-8, 2002, T2 (Abstract): 230.

18. Hongo RH, Ley J, Dick SE, Yee RR. The effect on clopidogrel in combination with aspirin when given before coronary artery bypass grafting. J Am Coll Card. 2002;40:231-7.

19. Yende S, Wunderfink RG. Effect of clopidogrel on bleeding after coronary bypass surgery. Crit Care Med. 2001;29:1271-5. 